\title{
Bilateral retinal artery occlusion due to mitral valve prolapse
}

\author{
F van Rhee, T E Blecher, K A DeLepeleire, N R Galloway
}

\begin{abstract}
We report a case of bilateral retinal artery occlusion due to mitral valve prolapse. Most patients with retinal ischaemia in whom it is found have not been previously known to have it. Since it is a common condition it would seem essential that it be included in the differential diagnosis of amaurosis fugax and retinal artery occlusion if future ischaemic events are to be prevented. All patients with retinal ischaemia should have a full cardiovascular examination supplemented by echocardiography.
\end{abstract}

Several cardiovascular causes of retinal ischaemia have been described including rheumatic valvular heart disease, calcified aortic disease, subacute bacterial endocarditis, valvular prostheses, cardiac myxomata, and carotid artery atherosclerosis. Mitral valve prolapse (MVP) is the commonest form of valvular heart disease in adults, and it has been increasingly recognised that it is associated with thromboembolism, including embolic phenomena in the ophthalmic circulation. MVP is easily missed, since these patients may have few cardiac symptoms or physical signs. Patients with it may first present with amaurosis fugax or rarely retinal artery occlusion, and awareness of the disease is therefore important to the ophthalmologist. We describe here a patient with bilateral retinal artery occlusion due to idiopathic prolapse of the mitral valve.

\section{Case report}

A 69-year-old woman presented with sudden painless loss of vision of the right eye followed six hours later by diminished vision in the inferonasal field of the left eye. There was no previous history of transient ischaemic attacks, amaurosis fugax, or rheumatic fever. She denied having had chest pain, dyspnoea, or palpitations. Essential thrombocythaemia had been diagnosed 10 years previously and was successfully treated with a single dose of radioactive phosphorus. Her platelet count had remained normal since.

Ocular examination showed a dense relative right afferent pupil defect with no light perception in the right eye and 6/9 vision in the left. Funduscopy revealed occlusion of the central retinal artery in the right eye due to a white refractile embolus lodged at the disc centre. Severe retinal oedema with a cherry red spot in the macula and supramacular haemorrhages were noted, while the peripheral retina had a normal non-ischaemic appearance (Fig 1). The embolus at the disc did not glisten with arterial pulsation or change position. In the left fundus an embolus was present in one of the superior arteriolar branches, leading to retinal oedema confined to the superotemporal quadrant. Field examination to confrontation of the left eye confirmed an inferior field defect, and on the Amsler grid the patient could point out a dense inferior scotoma.

Physical examination revealed no evidence of hypertension, subacute bacterial endocarditis, or carotid artery bruits. However, a midsystolic click followed by a late systolic murmur was heard at the apex. Echocardiography confirmed the presence of thickened mitral valve leaflets, both of which prolapsed during systole (Fig 2). The left atrium was dilated, but no thrombus was found (Fig 3). A 12-lead electrocardiogram was normal, but 24-hour electrocardiographic monitoring detected brief (less than 9 seconds) asymptomatic episodes of atrial fibrillation (150 beats per minute). Ultrasonography and Doppler studies of the neck revealed no significant atheromatous disease of the carotid arteries. Laboratory investigations showed $\mathrm{Hb} 14.5 \mathrm{~g} / \mathrm{dl}$, leucocytes $9.5 \times 10^{9} / \mathrm{h}$, and platelets $400 \times 10^{9} / 1$. The erythrocyte sedimentation rate was $9 \mathrm{~mm}$ in the first hour. Further laboratory studies including urea and electrolytes, liver function tests, blood glucose, autoantibody screen, serum protein electrophoresis, platelet function tests, triglycerides, and cholesterol gave results within normal limits.

Treatment was instituted with warfarin and aspirin. Her visual acuity has not improved, but she has so far had no further episodes of cerebral or retinal ischaemia.

\section{Discussion}

The incidence of MVP in the general population is $2.5 \%$ to $5 \% .^{1}$ Although it was initially thought to be a benign disorder, it has now been associated with subacute bacterial endocarditis, mitral

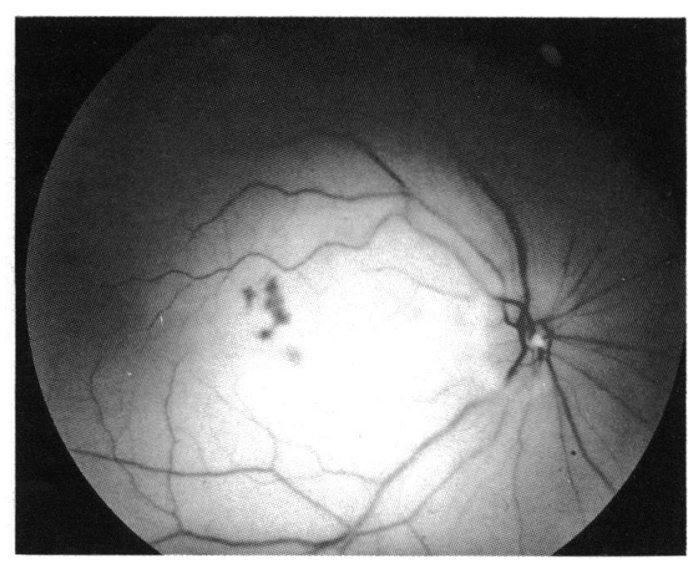

Figure 1: Refractile embolus lodged in right central retinal artery. Cherry red spot and supramacular haemorrhages noted. 


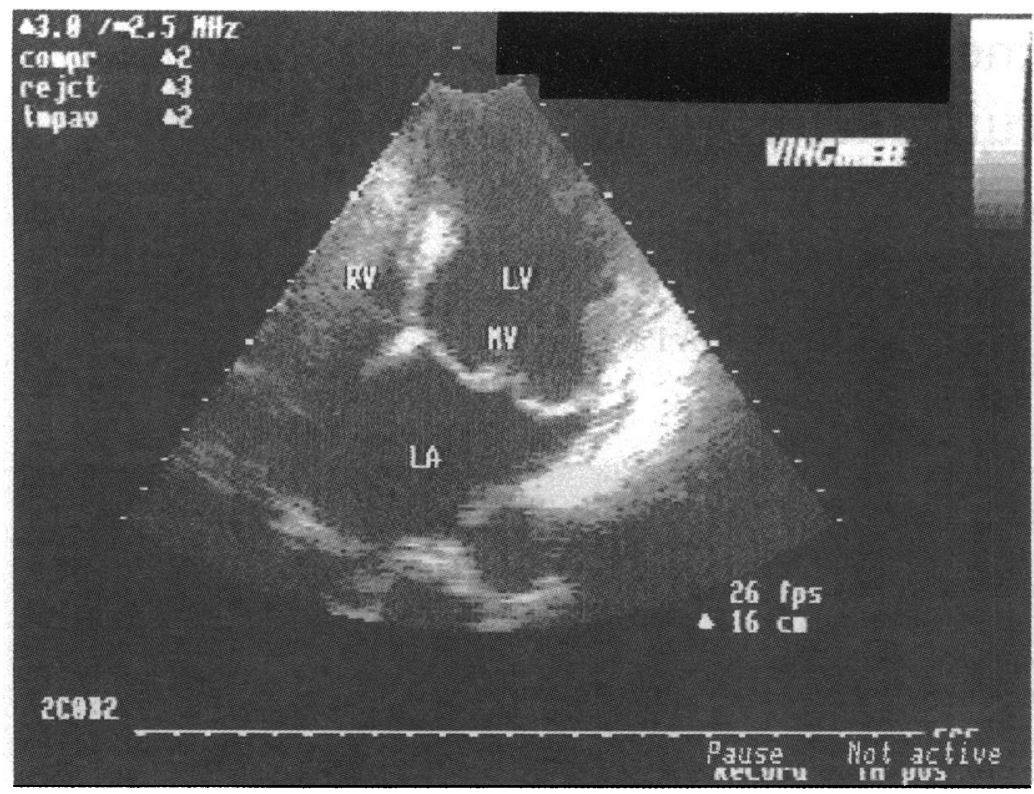

Figure 2: 2D -

Echocardiogram. Prolapse of both mitral valve cusps into the left atrium during systole. $L A=$ left atrium. $L V=$ left ventricle. $R V=$ right ventricle. $M V=$ mitral valve. regurgitation, atrial and ventricular dysrhythmias, and sudden death. Reported thromboembolic complications include transient ischaemic attacks, stroke, amaurosis fugax, and rarely retinal artery occlusion. ${ }^{2}$ Symptoms of MVP are atypical chest pain, palpitations, syncope, and dyspnoea. On auscultation a characteristic mid systolic click followed by a late systolic murmur may be heard, while sitting, standing, and the Valsalva manoeuvre all accentuate the murmur. ${ }^{3}$ Two-dimensional echocardiography confirms the diagnosis.

Our patient is unusual in having simultaneous bilateral retinal artery occlusion, which strongly suggests a cardiac origin of the emboli. Retinal examination revealed the typical calcific emboli associated with valvular heart disease and sudden permanent loss of vision. ${ }^{4}$ Visual and neurological dysfunction in essential thrombocythaemia is associated with the presence of thrombocytosis and spontaneous platelet aggregation. Since both platelet count and platelet function were normal, it is unlikely that essential thrombocythaemia should be implicated as the cause of her emboli. ${ }^{5}$ Ultrasonography and Doppler flow studies of both carotid arteries gave no evidence of atheromatous plaques as a source of emboli.

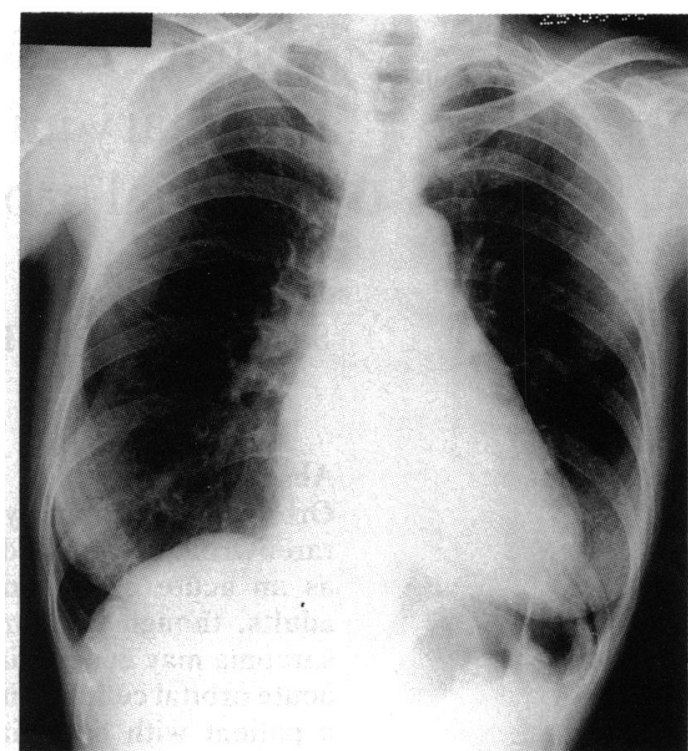

Figure 3: Chest $x$ ray showing bulging of the left heart border due to enlargement of the left atrium.

Most patients with cerebral or retinal ischaemic attacks in whom MVP is found have not been previously known to have MVP. ${ }^{6}$ The cardiovascular examination may be entirely normal and the history negative. ${ }^{7}$ These patients may first present to the ophthalmologist, and it is important that MVP should be included in the differential diagnosis of amaurosis fugax and retinal artery occlusion if further thromboembolic episodes are to be prevented. All patients presenting with retinal ischaemia should have a thorough cardiovascular assessment followed by echocardiography.

1 Levy D, Savage D. Prevalence and clinical features of mitral valve prolapse. Am Heart f 1987; 113: 1281-90

2 Barnett HJM, Boughner DR, Taylor DW, Cooper PE, Kostuk WJ, Nichol PM. Further evidence relating mitral valve prolapse to cerebral ischaemic events. $N$ Engl $\mathcal{F}$ Med 1980; 302: $139-44$

3 Devereux RB, Perloff JK, Reichek N, Josephson ME. Mitral valve prolapse. Circulation 1976; 54: 3-14.

4 Young BR. The significance of retinal emboli. 7 Clin Neuro Ophthalmol 1989; 9: 190-4.

5 Preston FE, Martin JF, Stewart RM, Davies-Jones GAB. Thrombocytosis, circulating platelet aggregates, and neurological dysfunction. BrMed 7 1979; ii: 1561-3.

6 Barnett HJM. Embolism in mitral valve prolapse. Annu Rev Med 1982; 33: 489-507.

7 Wilson LA, Keeling PWN, Malcolm AD, Russell RWR, Webb-Peploe MM. Visual complications of mitral leaflet prolapse. BrMed F 1977; ii: 86-8. 